\title{
Ex vivo propagation in a novel 3D high-throughput co-culture system for multiple myeloma
}

\author{
Johannes M. Waldschmidtt ${ }^{1,2}$ (D) Stefan J. Fruttiger ${ }^{1,3} \cdot$ Dagmar Wider $^{1} \cdot$ Johannes Jung $^{1,2} \cdot$ Andreas R. Thomsen $^{4}$. \\ Tanja N. Hartmann ${ }^{1,2} \cdot$ Justus Duyster ${ }^{1,2} \cdot$ Martin J. Hug ${ }^{3} \cdot$ Kareem A. Azab $^{6} \cdot$ Manfred Jung $^{5} \cdot$ Ralph Wäsch ${ }^{1,2}$. \\ Monika Engelhardt ${ }^{1,2}$
}

Received: 13 July 2021 / Accepted: 4 November 2021 / Published online: 24 January 2022

(c) The Author(s) 2022

\begin{abstract}
Purpose Multiple myeloma (MM) remains an incurable hematologic malignancy which ultimately develops drug resistance and evades treatment. Despite substantial therapeutic advances over the past years, the clinical failure rate of preclinically promising anti-MM drugs remains substantial. More realistic in vitro models are thus required to better predict clinical efficacy of a preclinically active compound.

Methods Here, we report on the establishment of a conical agarose 3D co-culture platform for the preclinical propagation of primary MM cells ex vivo. Cell growth was compared to yet established 2D and liquid overlay systems. MM cell lines (MMCL: RPMI-8226, U266, OPM-2) and primary patient specimens were tested. Drug sensitivity was examined by exploring the cytotoxic effect of bortezomib and the deubiquitinase inhibitor auranofin under various conditions.

Results In contrast to 2D and liquid overlay, cell proliferation in the 3D array followed a sigmoidal curve characterized by an initial growth delay but more durable proliferation of MMCL over 12 days of culture. Primary MM specimens did not expand in ex vivo monoculture, but required co-culture support by a human stromal cell line (HS-5, MSP-1). HS-5 induced a $>$ fivefold increase in cluster volume and maintained long-term viability of primary MM cells for up to 21 days. Bortezomib and auranofin induced less cytotoxicity under 3D vs. 2D condition and in co- vs. monoculture, respectively.

Conclusions This study introduces a novel model that is capable of long-term propagation and drug testing of primary MM specimens ex vivo overcoming some of the pitfalls of currently available in vitro models.
\end{abstract}

Keywords Multiple myeloma $\cdot$ Drug discovery $\cdot$ In vitro modeling $\cdot$ Bone marrow microenvironment $\cdot$ Bortezomib . Auranofin

Parts of the results were presented at the 'American Society of Hematology' (ASH) 2017, and at the 'German, Austrian and Swiss Hematology \& Oncology’ (DGHO) meetings in 2016-2018.

Monika Engelhardt

monika.engelhardt@uniklinik-freiburg.de

1 Department of Internal Medicine I, Faculty of Medicine and Medical Center, University of Freiburg, Hugstetterstr. 53, 79106 Freiburg, Germany

2 Comprehensive Cancer Center Freiburg (CCCF), Freiburg University Medical Center, Freiburg, Germany

3 Pharmacy, Freiburg University Medical Center, Freiburg, Germany

\section{Introduction}

Multiple myeloma (MM) is a plasma cell disorder with an expected number of 34,920 new cases in the United States in 2021 (American Cancer Society 2021). It is characterized by end-organ damage, such as anemia, hypocalcemia,

4 Department of Radiation Oncology, Freiburg University Medical Center, Freiburg, Germany

5 Institute of Pharmaceutical Sciences, University of Freiburg, Freiburg, Germany

6 Department of Radiation Oncology, Washington University, St. Louis, MO, USA 
renal insufficiency, and bone lesions (Kyle and Rajkumar 2009). Overall response rates to induction therapy have substantially improved over the past decades and are nowadays consistently $>90 \%$, with a pooled 5 year overall survival (OS) of 54\% across all age and risk groups (American Cancer Society 2019). Despite such substantial progress, MM remains an incurable disease that ultimately develops resistance and evades treatment. Novel therapeutic strategies are thus needed, especially for patients with triple-refractory $\mathrm{MM}$, i.e., a disease state that is refractory to bortezomib, lenalidomide, and anti-CD38 antibodies (Varga et al. 2020). The failure rate of clinical trials, however, remains sizeable as exemplified by the limited clinical activity of the antiIL-6 antibody siltuximab (Voorhees et al. 2013), the antiBAFF antibody tabalumab (Raje et al. 2016, 2017), and the anti-CXCR4 antibody ulocuplumab (Ghobrial et al. 2020) in patients with relapsed/ refractory MM (RRMM). More realistic preclinical models are thus urgently required to better predict later clinical success in patients.

In this study, we report on the establishment of a threedimensional (3D) co-culture platform for the preclinical propagation of primary MM cells ex vivo. As a basis for our model, we used a conical agarose 3D microwell array (3D CoSeedis ${ }^{\mathrm{TM}}$ ) which so far has been developed for solid tumors (Thomsen et al. 2017). MM cells are cultured in conical microwells formed of a non-adherent agarose matrix with bone marrow (BM)-derived stromal cells plated on the base of each plate. This distant co-culture model allows for the diffusion of soluble cytokines between BM stromal and MM cells. It stimulates aggregate formation of primary patient cells and allows for co-culture with compounds of the BM stroma for up to 21 days ex vivo. To examine the applicability of our model, we exemplified drug testing in comparison to yet established in vitro models by assessing the cytotoxic efficacy of the proteasome inhibitor bortezomib under 3D vs. $2 \mathrm{D}$ conditions and in co- vs. monoculture. We recapitulated our findings by testing auranofin, an inhibitor of proteasomeassociated deubiquitinases (DUB) with reported preclinical activity in MM (Nakaya et al. 2011).

In addition to reducing cost and time for more realistic preclinical drug screening, the here established 3D model holds the potential to perform drug testing in primary patient samples and to predict individual treatment responses ex vivo in real-time.

\section{Methods}

\section{Tumor cell lines}

The MM cell lines (MMCL) RPMI-8226, U266, OPM-2, and the human stromal cell line HS-5 were obtained from commercial sources (DSMZ, Braunschweig, Germany).
MSP-1 was provided by Dr. K. Azab (Washington University, St. Louis, MO) (de la Puente et al. 2016). All cell lines were cultured as previously reported (Udi et al. 2013; Waldschmidt et al. 2017). Authentication of cell lines was performed using DNA fingerprinting with small tandem repeat (STR) profiling. All cell lines were tested and found to be negative for mycoplasma contamination.

\section{Primary samples}

BM samples from six patients with untreated or relapsed/ refractory MM were included in this study. The mean BM infiltration rate at the time of sample acquisition was $83 \%$ (range 80-90\%). All patients provided written informed consent in accordance with local ethical standards (UKF protocol 212-16) and the Declaration of Helsinki. Mononuclear cells were isolated by density gradient centrifugation (Ficoll), frozen, and stored in liquid nitrogen until further use. Given the high percentage of histologically determined BM infiltration rates at the time of sample acquisition, thawed MM cells were further enriched using MACS technology-based bead selection for CD117 (Miltenyi Biotec). This approach helped to avoid interference with therapeutically relevant antigens (CD38, SLAMF7, and BCMA) (Schmidt-Hieber et al. 2011) and was independent of the well-described phenomenon of CD138 downregulation after storage at $-80^{\circ} \mathrm{C}$ (Frigyesi et al. 2014). To allow for comparability, a density of $5 \times 10^{5}$ cells per $\mathrm{mL}$ was used in all experiments for primary MM cells.

\section{D CoSeedis ${ }^{\mathrm{Tm}}$ conical agarose microwell array}

3D CoSeedis ${ }^{\mathrm{TM}}$ (abc biopply) is an agarose-based microwell device manufactured by computer-aided design (Fig. 1A) (Thomsen et al. 2017). The device is disk-shaped and contains conical microwells at a periodicity of 1 or $2 \mathrm{~mm}$ for 6- and 24-well plates, respectively (Fig. 1B). To monitor cell growth, plates were scanned on a high-resolution transmitted-light scanner (CanoScan 9000F Mark II) at $1200 \mathrm{dpi}$ (Fig. 1C, D). From the central portion of each scan, an area of $4 \mathrm{~cm}^{2}$ comprising 100 or 400 microwells was cropped. Each aggregate was measured using ImageJ processing software (National Institute of Health). Quantification of cytokines was performed using the LUNARIS ${ }^{\text {TM }}$ Human Multi-Plex Cytokine Kit (Ayoxxa Biosystems).

\section{Liquid overlay model}

Liquid overlay refers to a cell culture model that allows for the reproducible formation of spheroidal cell clusters 

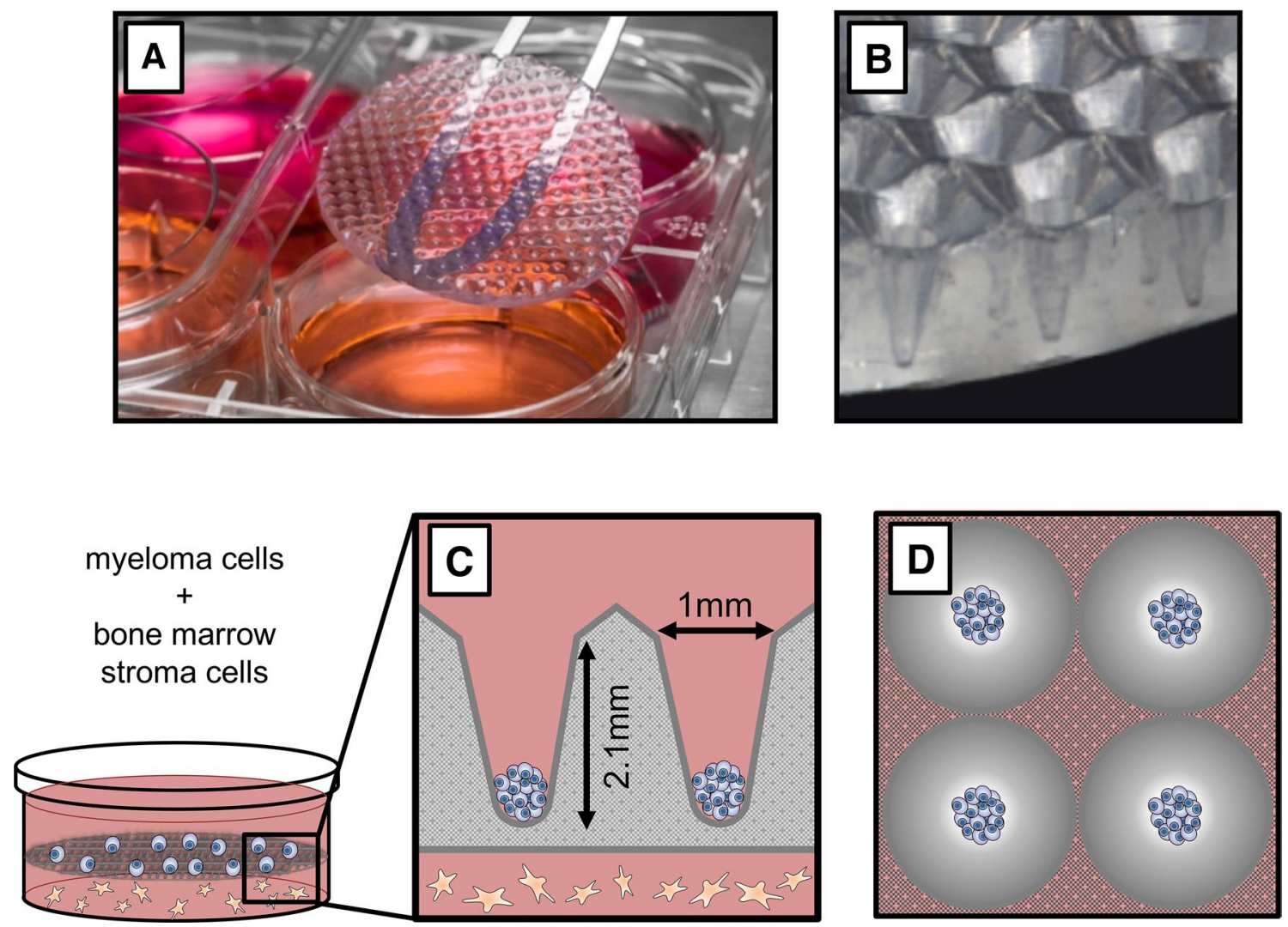

Fig. 1 Details of the conical agarose microwell array platform. A Photography of the agarose microwell disk inserted into a 6-well plate. B Lateral view showing conical microwells inside a membrane. C Sketch depicting distance co-culture. MM cells are seeded into 3D
CoSeedis $^{\mathrm{TM}}$, and then, the microwell array is placed on the monolayer composed of BM stromal cells. Agarose permeability allows for diffusion of gas and small biomolecules. Each cavity measures $1 \times 2.1 \mathrm{~mm}$. D Sketch showing cell aggregates in four microwells

\section{Confocal microscopy}

3D microarrays containing mCherry-transduced RPMI-8226 were stained with equal amounts of CellTrace CFSE or CellTrace Violet $(1.5 \mu \mathrm{l})$ for $30 \mathrm{~min}$ and washed twice. Confocal microscopy was performed on an LSM 880 Airyscan (Zeiss Microsystems).

\section{IHC staining}

Microwell devices were incubated in 2\% formalin solution overnight and sealed until further processing. Embedding was performed using $2.4 \%$ low melting point agarose. Following dehydration and paraffin embedding, $2 \mu \mathrm{m}$-thick sections were cut and mounted onto slides. Antibodies used for staining are displayed in Table S1.

\section{Cytotoxicity analysis}

A total of $2 \mathrm{ml}$ of HS-5 stroma cells were plated out with a density of $1 \times 10^{5}$ cells per $\mathrm{mL}$ on the bottom of a 6 -well for cells expressing histone H2B-mCherry. 
plate. To equilibrate the CoSeedis ${ }^{\mathrm{TM}}$ matrix, the scaffold was set on top of the stroma layer for 2-3 h. After $24 \mathrm{~h}$, media was replaced by $9 \mathrm{~mL}$ of MMCL-containing cell suspension $\left(5 \times 10^{5}\right.$ cells per $\left.\mathrm{mL}\right)$. Cells settled for another $24 \mathrm{~h}$ before either DMSO-diluted bortezomib (Selleck Chemicals) or auranofin (Santa Cruz) was added. After $48 \mathrm{~h}$ of drug exposure, cell viability was estimated using the CellTiter-Glo assay (Promega).

\section{Flow cytometry}

Adherent cells were detached using trypsin-EDTA and transferred to filter tubes. In $3 \mathrm{ml}$ fresh and cold PBS, cells were washed and centrifuged at $1200 \mathrm{rpm}$ over $8 \mathrm{~min}$. Pellets were dissolved and stained in $200 \mu \mathrm{L}$ staining solution with antibodies as indicated in Table S1. $1 \times 10^{5}$ cells were measured per condition on an FACS Calibur (Becton Dickinson). Analysis was performed using CellQuest ${ }^{\mathrm{TM}}$ Pro, FACS Diva (version 8.0.1) and FlowJo (version 10.3).

\section{Results}

\section{D microwell design allows for aggregate formation and recapitulates $B M$ support}

To compare and optimize ex vivo propagation of MM cells, RPMI-8226 cells were cultured in three different in vitro platforms. These platforms included a conventional 2D monolayer model, a liquid overlay approach, as well as the culture of cells in a novel 3D microwell device that allows for self-assembly of cell clusters (Figs. 1A-D, 2A). Aggregate formation in the latter model was additionally supported by a centrifugation step which forced seeding of MMCL into the cavities at an angle of $20^{\circ}$. Cell harvesting was performed by spinning the flipped matrices at a speed of $500 \mathrm{rpm}$. Cell proliferation in the monolayer and liquid overlay (measured as ATP content) followed bell-shaped kinetics with a maximal growth at $\sim$ day 6 , followed by a decay and full apoptosis at $\sim$ day 10 (Fig. 2B). Cell proliferation in the microwell model corresponded to a sigmoidal curve, i.e., the initial growth was delayed but developed more durably and was ongoing after 12 days of observation. Since RPMI8226 cells grow mostly in suspension, but may occasionally adhere and form clusters, microscopical inspection was performed throughout the experiment. Neither the monolayer nor the liquid overlay model produced cluster formation, whereas the cells in the microwells started to assemble and form instant cell aggregates within the conical microwells. As a second readout of cell proliferation, quantification of cell aggregate volumes over time was performed by a transmitted-light scanner approach (Thomsen et al. 2017). The proliferation rates determined by this approach faithfully recapitulated the data gained by the ATP-based quantification method (Fig. 3A, B). Both ATP quantification and transmitted-light scanner method revealed a slower proliferation rate for RPMI-8226 in the presence of the human BM stromal cell line compared to monoculture, which may point to a delayed but more durable establishment of cell-cell communication by continuous cytokine secretion (Fig. 3A, B).

To determine the permeability of our approach for cytokines and therapeutics, RPMI-8226 cells were transduced with the gene coding for the fluorescent protein mCherry followed by subsequent incubation with CellTrace CFSE and CellTrace Violet. Fluorescence was examined by confocal microscopy. Both dyes permeated the cell membrane of viable cells and penetrated to the core of each cell aggregate (Fig. 4A). The agarose hydrogel itself did not
A

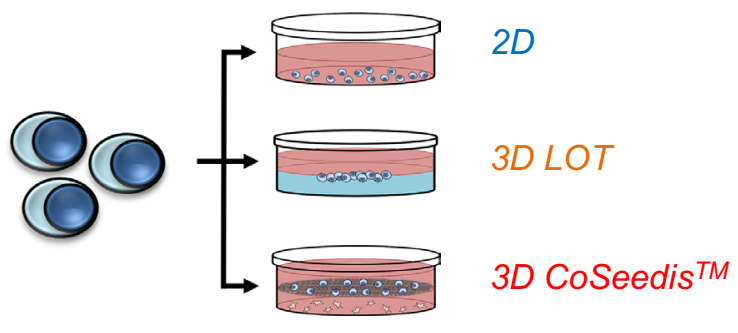

B Proliferation

CellTiter-Glo® (RPMI-8226, n=4)

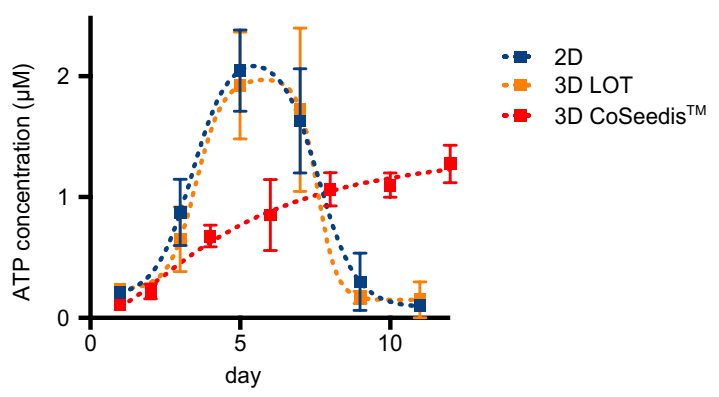

a bell-shaped growth pattern for RPMI-8226 cells in the 2D and LOT model. Cell growth in the 3D microwell model was delayed but followed a sigmoidal pattern and was ongoing after 12 days of observation 
Fig. 3 Impact of HS-5 stromal co-culture on proliferation and cluster size. A ATP content in RPMI-8226 cells culture in the $3 \mathrm{D}$ conical agarose microwell array as mono- vs. HS-5 coculture. B Cluster volume of RPMI-8226 cells culture in the 3D conical agarose microwell array as mono- vs. HS-5 co-culture measured by transmittedlight scanner method. C Sketch showing cell aggregates in three microwells. This model uses the measuring cup principle for determination of cell proliferation. D Microscopy and scan of agarose matrix disk containing cell aggregates $(100 \times, 25 \times$ and $1 \times)$
A Proliferation (RPMI-8226, $\mathrm{n}=4$ )

3D microwell model, CellTiter-Glo ${ }^{\circledR}$

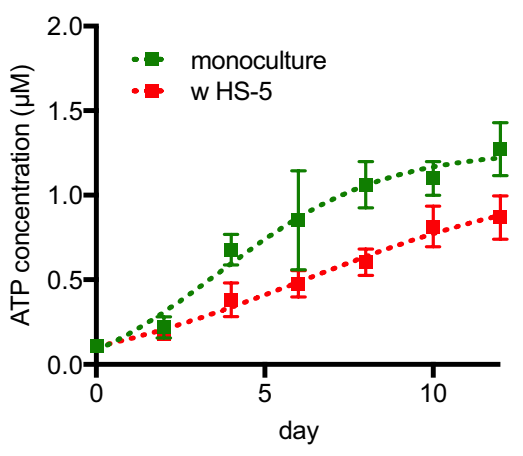

B Cell cluster growth (RPMI-8226, $n=6$ ) 3D microwell model, transmitted light scanner

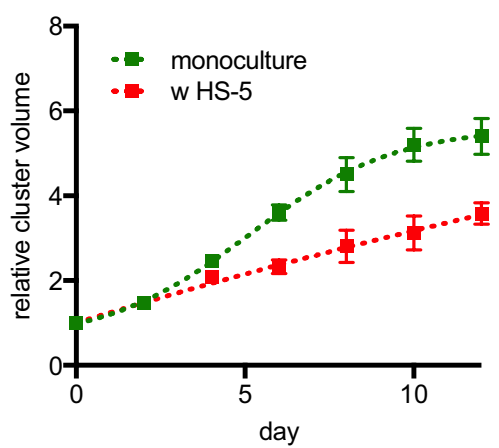

C Transmitted light scanner method Measuring cup principle

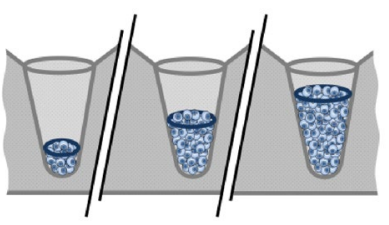

D Transmitted light scanner method Measurement of cluster volume

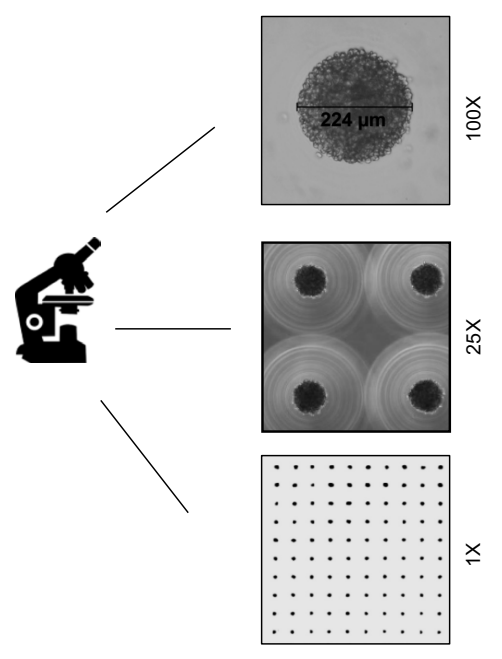

absorb any fluorescent dye, thereby permitting a transparent background for imaging analysis.

\section{Stromal co-culture and cluster formation foster resistance to bortezomib and auranofin}

Based on previous reports describing the critical role of stromal support for drug resistance in MM (Azab et al. 2009a, b, 2012; Waldschmidt et al. 2017), we hypothesized that the addition of a distant co-culture support would provide further benefit for MM cells within the microwell cavities and therefore tested the sensitivity of MM cells toward chemotherapy in this system.

After $48 \mathrm{~h}$, bortezomib-induced cytotoxicity in RPMI8226 cells was $\sim$ twofold lower in the 3D model as compared to the normal 2D flat culture (Fig. 4B), thereby demonstrating the impact of cluster formation on drug resistance of MM cells. Notably, sensitivity to bortezomib was even lower in the 3D model with HS-5 stromal support $(P \leq 0.01)$. To determine that reduced sensitivity to anti-MM drugs was not restricted to proteasome inhibitors, we recapitulated the impact of cluster formation and HS-5 stromal support by investigating the cytotoxic potency of the DUB inhibitor auranofin (AUR). AUR exemplifies a cytotoxic drug with potent in vitro activity in multiple cancers but so far limited efficacy in the clinic (Bonolo de Campos et al. 2020). As an inhibitor of the deubiquitinase system, AUR has reportedly high efficacy in diseases with a dysregulated ubiquitin-proteasome system (UPS), including MM (Nakaya et al. 2011; Raninga et al. 2015; Wang et al. 2019; Sze et al. 2020). The sensitivity to AUR in each model was exemplified by exposing MM cells to $3 \mu \mathrm{M}$ AUR. Cytotoxicity after $48 \mathrm{~h}$ of AUR treatment was reduced by $\sim 1.5$-fold in RPMI- 8226 cells treated within the 3D model as compared to cells treated within the normal 2D flat culture (Fig. 4D). Sensitivity to AUR was even lower in the 3D model with HS-5 stromal support $(P \leq 0.001)$. To determine that reduced sensitivity to AUR was not restricted to RPMI-8226, we recapitulated the impact of cluster formation and HS-5 stromal support by investigating the sensitivity to auranofin (AUR) in U266 as a second MMCL (Figure S1A) (Bonolo de Campos et al. 2020). CD138 positivity correlated with the cytotoxic potency of both drugs (Fig. 4C, E, S1B), with untreated cells showing significantly higher CD138 expression in the 3D vs. 2D setting. This observation may be critical for future phenotypic analyses as a more realistic approximation of 
A Microwell drug penetration RPMI-8226, confocal microscopy $100 \mathrm{X}$

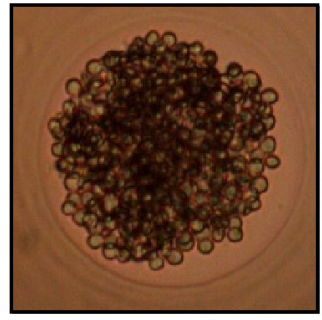

in culture

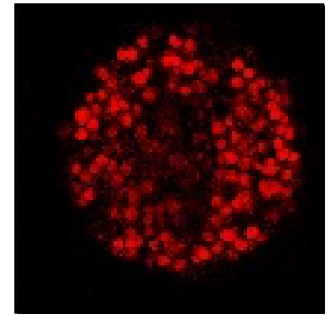

mCherry

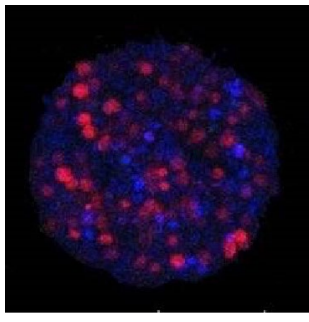

CellTrace Violet

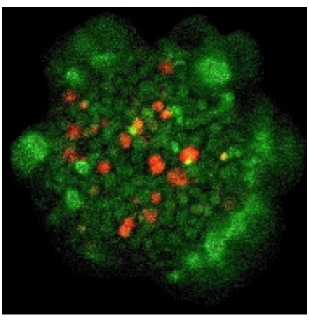

CFSE
B PI staining RPMI-8226, n=5, 6nM BTZ (48h)

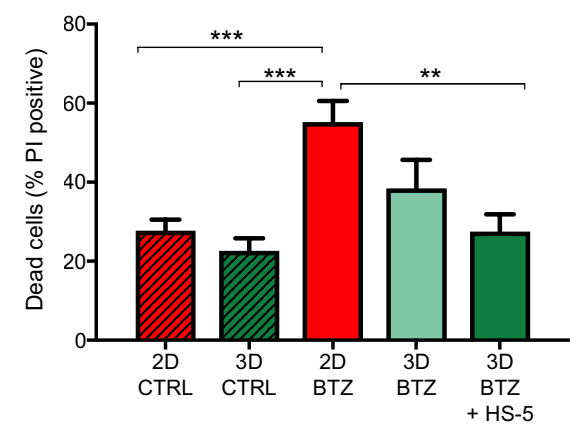

D PI staining

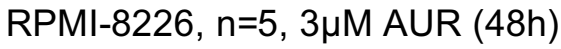

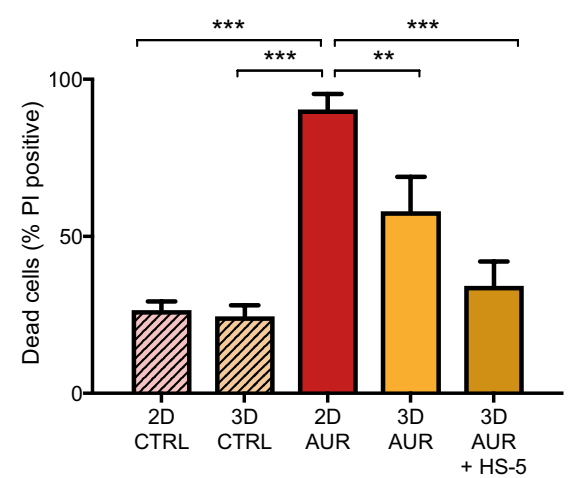

Fig. 4 Drug resistance in the conical agarose microwell array. A Microscopy (left panel) and confocal microscopy of one microwell show equal distribution of CFSE (green) and CellTrace Violet within an aggregate of mCherry-transduced RPMI-8226. B Comparison of PI positivity in untreated RPMI-8226 cells and RPMI8226 cells treated with bortezomib $(6 \mathrm{nM})$ over $48 \mathrm{~h}$. C Comparison of CD138 positivity in untreated RPMI-8226 cells and RPMI-8226

\section{CD138 positivity RPMI-8226, n=8, 6nM BTZ (48h)}

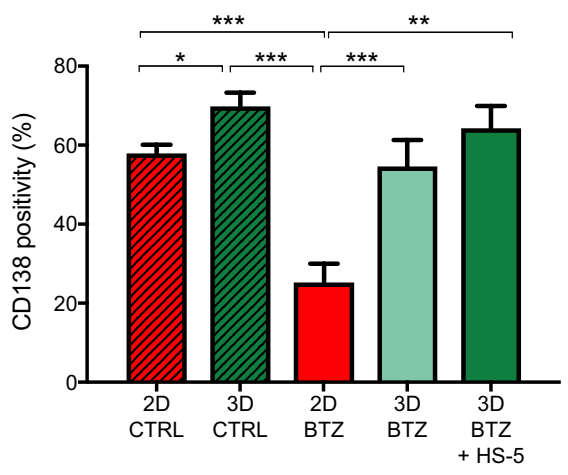

\section{E CD138 positivity

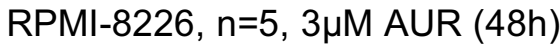

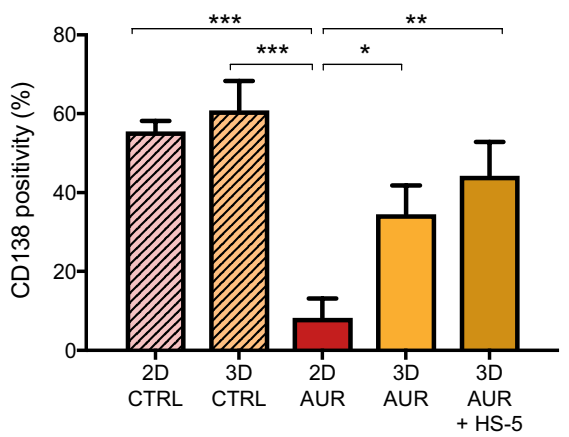

cells treated with bortezomib (6 $\mathrm{nM})$ over $48 \mathrm{~h}$. D Comparison of PI positivity in untreated RPMI-8226 cells and RPMI-8226 cells treated with auranofin $(3 \mu \mathrm{M})$ over $48 \mathrm{~h}$. E Comparison of CD138 positivity in untreated RPMI-8226 cells and RPMI-8226 cells treated with auranofin $(3 \mu \mathrm{M})$ over $48 \mathrm{~h}$. Conditions in B-E varied by model (2D vs. $3 \mathrm{D}$ microwell) and co-culture (monoculture vs. HS-5 co-culture). $\mathrm{P}$ values are as indicated $* P \leq 0.05, * * P \leq 0.01, * * * P \leq 0.001$ 
antigen expression ex vivo may impact the evaluation of therapeutically relevant antigen expression.

In summary, our data propose a need to incorporate stromal co-culture into ex vivo modeling and demonstrate the superiority of our 3D versus $2 \mathrm{D}$ approaches.

\section{Propagation of primary MM cells is reproducible ex vivo}

To evaluate the applicability of our 3D microwell model for ex vivo propagation of even more relevant primary MM cells, we measured cytokine concentrations in the respective supernatants after 6 days of culture using RPMI-8226, OPM-2, and primary MM cells. We observed increased secretion of IL1-beta, IL-2, IL-6, IL-8, and TNF-alpha, but reduced VEGF production in the presence of HS-5 stromal cells (Fig. 5A, Table S2).
Cell growth for MM cells from primary BM aspirates was next assessed in the presence or absence of stromal cell support. Primary MM cells were derived from the BM of six RRMM patients with reported BM infiltration of CD138 + / CD38 + plasma cells $\geq 80 \%$ (Table 1). Growth monitoring over time revealed that primary MM cells in monoculture did not expand and showed a moderate linear decline in cell volume over the first 21 days of culture (Fig. 5B). Co-culture with MSP-1, an MM-derived human stromal cell line, induced a sigmoidal expansion, characterized by a $>$ twofold increase in cell volume at day 21 of culture. HS-5 co-culture induced an even more beneficial increase in cell volume by $>5.5$-fold after 3 weeks. Viability was continuously assessed and confirmed by microscopy, panchromatic Pappenheim staining, and immunohistochemical (IHC) staining for CD38 (Fig. 5C).
A Cytokine secretion (day 6) mono- vs. HS-5 co-culture (\% normalized)

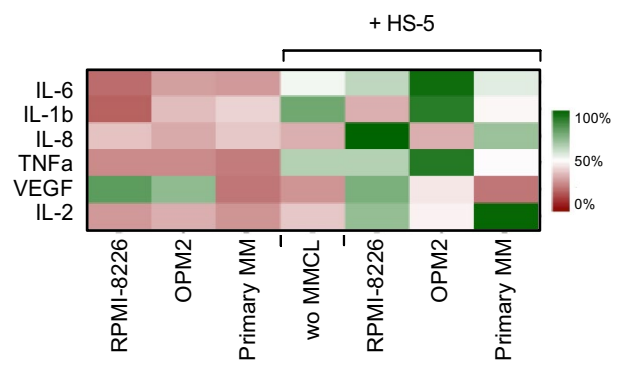

B Proliferation (pt specimens, $\mathrm{n}=6$ ) 3D microwell model, transmitted light scanner

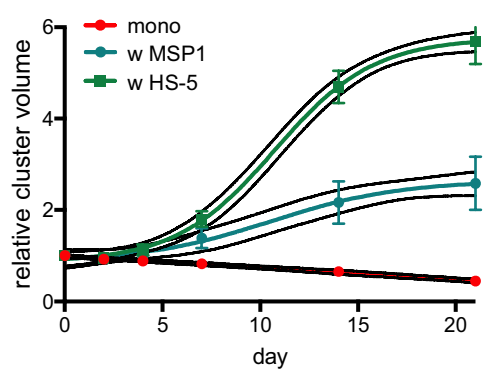

Fig. 5 Long-term propagation of primary MM cells ex vivo. A Cytokine secretion measured by multiplex array from supernatants of RPMI-8226, OPM-2, and primary MM cells after 6 days with and without HS-5 co-culture. B Cluster volume measured by transmittedlight scanner methods shows the critical impact of co-culture for the propagation of primary patient specimens ex vivo $(n=6)$. Growth support is improved with HS-5 as compared to MSP-1. Cells continue to proliferate at day 21 ex vivo. C Longitudinal monitoring of primary MM cells derived from the bone marrow of a 51-year-old patient with high-risk IgG kappa MM. Assessment is shown for day
Multimodal viability assessment (d14)
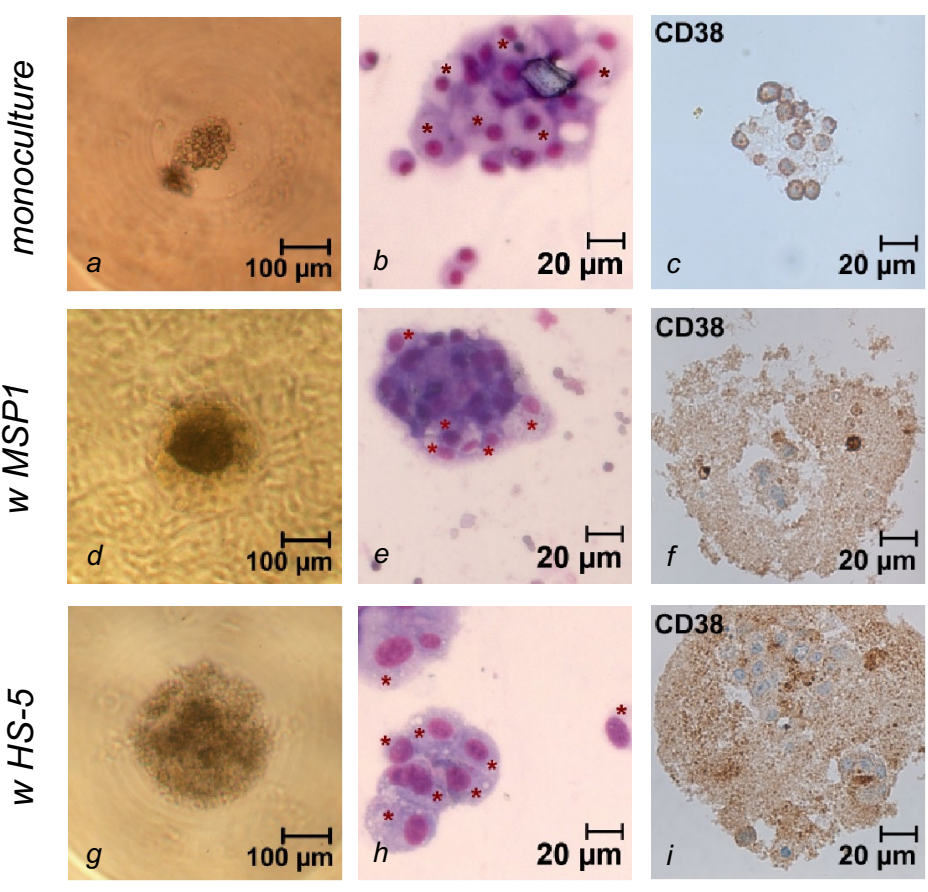

in culture

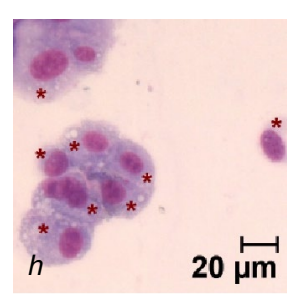

Pappenheim stain

14 ex vivo using microscopy (left column), Pappenheim stain (middle panel) and IHC for CD38 (right panel). Serial monitoring demonstrated less cluster cell expansion of MM cells in monoculture (a). Plasma cell (PC) morphology via microscopy/Pappenheim stain remained apparent (b), but with few CD38 positive PCs in IHC (c). Co-culture with MSP-1 stimulated cluster expansion of primary PCs (d). PCs were densely accumulating (e) and led to much larger PC clusters with CD38 positive cells (f) in the presence of MSP-1 stromal support. Cluster size and CD38 positivity were further enhanced in $\mathbf{g - i}$ with HS-5 stromal support in 3D culture 
Table 1 Individual patient characteristics and summary

\begin{tabular}{lllllllll}
\hline$\#$ & MM subtype & BM infiltration & CG & Age (yrs) & Gender & Status & ISS & Comment \\
\hline 1 & IgA kappa & $80 \%$ & HR & 69 & Female & RRMM & 1 & MM \\
2 & IgG kappa & $80 \%$ & HR & 70 & Male & RRMM & 2 & MM \\
3 & kappa-LC & $80 \%$ & HR & 52 & Male & RRMM & 1 & PCL \\
4 & IgG kappa & $80 \%$ & HR & 83 & Male & ID & 3 & MM \\
5 & IgA kappa & $90 \%$ & HR & 66 & Male & RRMM & 3 & MM \\
6 & IgG kappa & $90 \%$ & HR & 51 & Male & ID & 3 & MM \\
\hline$\sum$ median & IgG $(n=3)$ & $80 \%(80-90 \%)$ & HR $(n=6)$ & $68(51-83)$ & Female $(n=1)$ & RRMM $(n=6)$ & $1(n=2)$ & MM $(n=5)$ \\
$($ (range) $)$ & IgA $(n=2)$ & & & & Male $(n=5)$ & ID $(n=2)$ & $2(n=1)$ & PCL $(n=1)$ \\
& kappa-LC $(n=1)$ & & & & & & $3(n=3)$ \\
\hline
\end{tabular}

$M M$ multiple myeloma, RRMM relapsed/refractory MM, ID initial diagnosis, $P C L$ plasma cell leukemia, $E M-M M$ extramedullary MM, $C G$ cytogenetics, $H R$ unfavorable cytogenetics defined as deletion $17 \mathrm{p}, \mathrm{t}(4 ; 14), \mathrm{t}(14 ; 16), \mathrm{t}(14 ; 20)$ or gain $1 \mathrm{q}$

This demonstrated that our 3D microwell array is a suitable model to enable the survival and long-term culture of primary MM specimens ex vivo.

\section{Discussion}

Treatment of MM has undergone substantial advances with the establishment of several FDA-approved agents with high clinical efficacy over the past decades. However, preclinical models for MM remain inefficient in predicting later clinical efficacy. Between 1961 and 2013, single agent activity has been reported for more than 400 preclinically tested compounds, but only ten of these agents could be validated in phase III trials and are nowadays approved treatment options for the clinical management of patients (Kortuem et al. 2014). Understanding preclinical modeling for MM may help to optimize the translation of in vitro findings into clinical use, but is unfortunately still limited by the inability to reproducibly propagate primary MM patient specimens ex vivo.

MM cells strongly depend on the interaction with their BM microenvironment (Azab et al. 2009b; Waldschmidt et al. 2017). Current in vitro models tend to overemphasize anti-MM effects as they typically do not simulate such interaction of MM cells with their protective niche. As our knowledge on MM and its microenvironment has dramatically increased over the last years, more complex in vitro models have been introduced, thereby providing a wider range of test systems from low- to high-throughput approaches (Schüler et al. 2013). These platforms include two-dimensional (2D) monolayers, bioreactors, scaffoldbased models, and liquid overlay cultures (Abe et al. 2004; Kirshner et al. 2008; Zdzisińska et al. 2009; Breslin and O'Driscoll 2013; Reagan et al. 2014; de la Puente et al. 2015; Jakubikova et al. 2016; Santo et al. 2017). 2D models allow for high-throughput drug screening, but do not adequately assess the interaction of MM cells with their environment. More sophisticated 3D co-culture systems have integrated additional components which permit evaluation of a broader range of therapeutic strategies, but increase the complexity and thereby limit the applicability of 3D models to smaller throughput series (Table S3) (Breslin and O'Driscoll 2013; Schüler et al. 2013; Santo et al. 2017).

While prior models have reported that most primary MM cells do not proliferate ex vivo (Drewinko et al. 1981; Robillard et al. 2005), we here report on a novel conical agarose 3D microwell platform for the reproducible culture of primary MM cells. Our agarose-based microwell design facilitates aggregate formation which in turn has a significant impact on the long-term viability of BM-derived primary MM cells ex vivo. We recapitulate that stromal co-culture is essential for in vitro modeling of drug resistance phenomena and adequate preclinical prediction of drug sensitivity. To examine the applicability of our model for ex vivo drug testing, we investigated the cytotoxic effect of bortezomib and the DUB inhibitor auranofin with reportedly high in vitro efficacy in primary MM samples (Bonolo de Campos et al. 2020). We show that while both bortezomib and auranofin were highly potent in the absence of stromal cell support, they induced less cytotoxicity if MM cells (i) form cell-cell aggregates, and (ii) receive cytokine support via HS-5 stromal co-culture. This finding in the 3D co-culture setting is in line with prior work from our group and others describing stroma-induced drug resistance under 2D co-culture conditions (Azab et al. 2009b; Waldschmidt et al. 2017). At the same time, our platform allows for substantially longer cultivation periods of primary patient material and for robust separation of target cell populations, this being highly relevant for drug screening and testing over more extended cultivation periods ( $>2-3$ days) as currently 
feasible with 2D culture systems (Zlei et al. 2007; Udi et al. 2013; Lorenz et al. 2016).

Primary MM cells benefitted from higher cytokine levels in the supernatant and could be maintained in culture up to a culture period of 21 days. We have previously shown that combined supplementation of cytokines (IL-6, IGF-1, CXCL12, Galectin-1, and IL-1a) has substantial impact on the growth of MM cells (Zlei et al. 2007). Here, we provide more detailed cytokine characterization in the 3D setting, and show that IL-6, IL- $1 \mathrm{~b}$, IL-8, and TNF $\alpha$ secretion seem to be stimulated by the interaction of MMCL and primary MM cells with their respective stromal co-culture partner. This observation is in line with Zdzisinska and colleagues who reported increased IL-11 and HGF secretion by MMderived mesenchymal stem cells (MSCs) in the 3D versus 2D co-culture setting (Zdzisińska et al. 2009). Other than expected, addition of HS-5 led to a decrease of VEGF in the co-culture supernatants. This appeared different to our current understanding on the role of neo-angiogenesis and VEGF signaling for MM cell propagation (Le Gouill et al. 2004; Neri et al. 2011) and most likely reflected the more delayed growth kinetics in our model. Studies with serial cytokine measurements over time are currently ongoing and will investigate if incorporation of other cell types, most critically endothelial cells, may provide an even better cytokine environment for the long-term propagation of primary MM cells (Podar and Anderson 2007).

Aside from our platform, other in vitro models have been developed with the intent to better recapitulate the MM-BM niche: osteoclasts, murine stromal cell lines, immature dendritic cells, and mesenchymal stem cells have all proven advantageous to stimulate proliferation of MM cells in vitro (Chauhan et al. 1996; Abe et al. 2004; Li et al. 2008; Kirshner et al. 2008; Azab et al. 2012). Major challenges of these currently available co-culture platforms, however, remain to (i) guarantee reproducible growth characteristics and (ii) avoid long-term phenotypic modulation attributable to the models themselves (Zlei et al. 2007; Yaccoby 2010; Udi et al. 2013).

By providing a 3D scaffold with preformed cavities, our model allows for the reproducible ex vivo propagation of MMCL and especially primary MM cells. This platform incorporates co-culture partners from the $\mathrm{BM}$ niche which seems critical to realistically determine the efficacy of future therapeutic compounds. Although it remains to be seen whether 3D models can substitute conventional testing systems, they may open up an entirely new frame for the in vitro assessment of anti-MM targets (Schüler et al. 2013). From our current understanding, we propose that $2 \mathrm{D}$ and $3 \mathrm{D}$ co-culture models should ideally complement each other with $2 \mathrm{D}$ being used for short-term $(<2-3$ days) assessment of MMCL in vitro and 3D approaches to be preferred for ex vivo cultivation of primary material over longer periods.

In summary, we here report on a novel 3D in vitro platform for the preclinical propagation of primary MM cells. While the readout of our system focuses primarily on morphological changes, cell viability, and cell growth kinetics, it also allows for the evaluation of surface marker expression, migration, and target expression of various anti-MM agents. From a clinical viewpoint, this model may improve our ability to better predict later clinical success of preclinically active compounds to reduce time and financial expenses of drug discovery for MM at the earliest stage possible.

Supplementary Information The online version contains supplementary material available at https://doi.org/10.1007/s00432-021-03854-6.

Acknowledgements The authors thank all MM patients and families who engaged in this study. We are grateful to Julia Felthaus and Christine Aldrian for valuable input. We are also thankful to all DSMM, GMMG, EMN, IMWG, and ASH experts who provided advice and discussion on the here presented data.

Author contributions SJF, JMW, DW, JF, and JS designed and performed experiments. SJF, JMW, DW, RW, and ME analyzed the data. JMW, SJM, DW, RW, and ME wrote the manuscript. JS, MJ, and MH provided pharmacodynamic expertise and provided insight for all HDAC affinity studies. ART, KAA, ML, and JD provided analytical support. JMW, SJF, MJ, RW, and ME designed the experimental strategy and supervised the analysis. All authors discussed the results and implications and reviewed the manuscript.

Funding Open Access funding enabled and organized by Projekt DEAL. No disclosures related to this publication.

Availability of data and materials Not applicable.

Code availability Not applicable.

\section{Declarations}

Conflict of interest A.R.T.: Inventor of 3D CoSeedis ${ }^{\mathrm{TM}}$ (patent \#EP2917326A1 and \#WO2014072432A1); consultant to abc biopply. 3D CoSeedis ${ }^{\mathrm{TM}}$ is commercially licensed by abc biopply, Cham, Switzerland. All other authors report no conflict of interest with regard to this publication.

Ethics approval All procedures performed in this study were performed in accordance with the Declaration of Helsinki and the International Conference on Harmonization Good Clinical Practice. All patients provided written informed consent to allow the use of available biopsies for the analysis and research purposes of this study.

Consent to participate Informed consent was obtained from all individual participants included in the study.

Consent for publication All authors consent for the publication of this manuscript. 
Open Access This article is licensed under a Creative Commons Attribution 4.0 International License, which permits use, sharing, adaptation, distribution and reproduction in any medium or format, as long as you give appropriate credit to the original author(s) and the source, provide a link to the Creative Commons licence, and indicate if changes were made. The images or other third party material in this article are included in the article's Creative Commons licence, unless indicated otherwise in a credit line to the material. If material is not included in the article's Creative Commons licence and your intended use is not permitted by statutory regulation or exceeds the permitted use, you will need to obtain permission directly from the copyright holder. To view a copy of this licence, visit http://creativecommons.org/licenses/by/4.0/.

\section{References}

Abe M, Hiura K, Wilde J et al (2004) Osteoclasts enhance myeloma cell growth and survival via cell-cell contact: a vicious cycle between bone destruction and myeloma expansion. Blood 104:2484-2491. https://doi.org/10.1182/blood-2003-11-3839

American Cancer Society. Key statistics for multiple myeloma. https:// www.cancer.org/cancer/multiple-myeloma/about/key-statistics. html. Accessed 13 June 2021

Azab AK, Azab F, Blotta S et al (2009a) RhoA and Rac1 GTPases play major and differential roles in stromal cell-derived factor-1-induced cell adhesion and chemotaxis in multiple myeloma. Blood 114:619-629. https://doi.org/10.1182/blood-2009-01-199281

Azab AK, Runnels JM, Pitsillides C et al (2009b) CXCR4 inhibitor AMD3100 disrupts the interaction of multiple myeloma cells with the bone marrow microenvironment and enhances their sensitivity to therapy. Blood 113:4341-4351. https://doi.org/10.1182/ blood-2008-10-186668

Azab AK, Hu J, Quang P et al (2012) Hypoxia promotes dissemination of multiple myeloma through acquisition of epithelial to mesenchymal transition-like features. Blood 119:5782-5794. https://doi. org/10.1182/blood-2011-09-380410

Bonolo de Campos C, Meurice N, Petit JL et al (2020) "Direct to Drug" screening as a precision medicine tool in multiple myeloma. Blood Cancer J 10:54. https://doi.org/10.1038/s41408-020-0320-7

Breslin S, O'Driscoll L (2013) Three-dimensional cell culture: the missing link in drug discovery. Drug Discov Today 18:240-249. https://doi.org/10.1016/j.drudis.2012.10.003

Chauhan D, Uchiyama H, Akbarali Y et al (1996) Multiple myeloma cell adhesion-induced interleukin- 6 expression in bone marrow stromal cells involves activation of NF-kappa B. Blood 87:1104-1112

Costa EC, Gaspar VM, Coutinho P, Correia IJ (2014) Optimization of liquid overlay technique to formulate heterogenic $3 \mathrm{D}$ co-cultures models. Biotechnol Bioeng 111:1672-1685. https://doi.org/10. 1002/bit. 25210

de la Puente P, Muz B, Gilson RC et al (2015) 3D tissue-engineered bone marrow as a novel model to study pathophysiology and drug resistance in multiple myeloma. Biomaterials 73:70-84. https:// doi.org/10.1016/j.biomaterials.2015.09.017

de la Puente P, Quan N, Hoo RS et al (2016) Newly established myeloma-derived stromal cell line MSP-1 supports multiple myeloma proliferation, migration, and adhesion and induces drug resistance more than normal-derived stroma. Haematologica 101:e307-311. https://doi.org/10.3324/haematol.2016.142190

Drewinko B, Alexanian R, Boyer H et al (1981) The growth fraction of human myeloma cells. Blood 57:333-338

Frigyesi I, Adolfsson J, Ali M et al (2014) Robust isolation of malignant plasma cells in multiple myeloma. Blood 123:1336-1340. https://doi.org/10.1182/blood-2013-09-529800
Ghobrial IM, Liu C-J, Redd RA et al (2020) A phase Ib/II trial of the first-in-class anti-CXCR4 antibody ulocuplumab in combination with lenalidomide or bortezomib plus dexamethasone in relapsed multiple myeloma. Clin Cancer Res 26:344-353. https://doi.org/ 10.1158/1078-0432.CCR-19-0647

Jakubikova J, Cholujova D, Hideshima T et al (2016) A novel 3D mesenchymal stem cell model of the multiple myeloma bone marrow niche: biologic and clinical applications. Oncotarget 7:7732677341. https://doi.org/10.18632/oncotarget.12643

Kirshner J, Thulien KJ, Martin LD et al (2008) A unique threedimensional model for evaluating the impact of therapy on multiple myeloma. Blood 112:2935-2945. https://doi.org/10.1182/ blood-2008-02-142430

Kortuem KM, Zidich K, Schuster SR et al (2014) Activity of 129 single-agent drugs in 228 phase I and II clinical trials in multiple myeloma. Clin Lymphoma Myeloma Leuk 14:284-290.e5. https:// doi.org/10.1016/j.clml.2013.12.015

Kyle RA, Rajkumar SV (2009) Criteria for diagnosis, staging, risk stratification and response assessment of multiple myeloma. Leukemia 23:3-9. https://doi.org/10.1038/leu.2008.291

Le Gouill S, Podar K, Amiot M et al (2004) VEGF induces Mcl-1 up-regulation and protects multiple myeloma cells against apoptosis. Blood 104:2886-2892. https://doi.org/10.1182/ blood-2004-05-1760

Li X, Pennisi A, Yaccoby S (2008) Role of decorin in the antimyeloma effects of osteoblasts. Blood 112:159-168. https://doi.org/ 10.1182/blood-2007-11-124164

Lorenz J, Waldschmidt J, Wider D et al (2016) From CLL to multiple myeloma - spleen tyrosine kinase (SYK) influences multiple myeloma cell survival and migration. Br J Haematol 174:985-989. https://doi.org/10.1111/bjh.13825

Nakaya A, Sagawa M, Muto A et al (2011) The gold compound auranofin induces apoptosis of human multiple myeloma cells through both down-regulation of STAT3 and inhibition of NF- $\mathrm{KB}$ activity. Leuk Res 35:243-249. https://doi.org/10.1016/j.leukres. 2010.05.011

Neri P, Ren L, Azab AK et al (2011) Integrin $\beta 7$-mediated regulation of multiple myeloma cell adhesion, migration, and invasion. Blood 117:6202-6213. https://doi.org/10.1182/blood-2010-06-292243

Podar K, Anderson KC (2007) Inhibition of VEGF signaling pathways in multiple myeloma and other malignancies. Cell Cycle 6:538-542. https://doi.org/10.4161/cc.6.5.3922

Raje NS, Faber EA, Richardson PG et al (2016) Phase 1 study of tabalumab, a human anti-B-cell activating factor antibody, and bortezomib in patients with relapsed/refractory multiple myeloma. Clin Cancer Res 22:5688-5695. https://doi.org/10.1158/10780432.CCR-16-0201

Raje NS, Moreau P, Terpos E et al (2017) Phase 2 study of tabalumab, a human anti-B-cell activating factor antibody, with bortezomib and dexamethasone in patients with previously treated multiple myeloma. Br J Haematol 176:783-795. https://doi.org/10.1111/ bjh. 14483

Raninga PV, Di Trapani G, Vuckovic S et al (2015) Inhibition of thioredoxin 1 leads to apoptosis in drug-resistant multiple myeloma. Oncotarget 6:15410-15424. https://doi.org/10.18632/oncotarget. 3795

Reagan MR, Mishima Y, Glavey SV et al (2014) Investigating osteogenic differentiation in multiple myeloma using a novel 3D bone marrow niche model. Blood 124:3250-3259. https://doi.org/10. 1182/blood-2014-02-558007

Robillard N, Pellat-Deceunynck C, Bataille R (2005) Phenotypic characterization of the human myeloma cell growth fraction. Blood 105:4845-4848. https://doi.org/10.1182/blood-2004-12-4700

Santo VE, Rebelo SP, Estrada MF et al (2017) Drug screening in 3D in vitro tumor models: overcoming current pitfalls of efficacy read-outs. Biotechnol J. https://doi.org/10.1002/biot.201600505 
Schmidt-Hieber M, Pérez-Andrés M, Paiva B et al (2011) CD117 expression in gammopathies is associated with an altered maturation of the myeloid and lymphoid hematopoietic cell compartments and favorable disease features. Haematologica 96:328-332. https://doi.org/10.3324/haematol.2010.031872

Schnerch D, Follo M, Felthaus J et al (2013) The 3' untranslated region of the cyclin B mRNA is not sufficient to enhance the synthesis of cyclin B during a mitotic block in human cells. PLoS ONE 8:e74379. https://doi.org/10.1371/journal.pone.0074379

Schüler J, Ewerth D, Waldschmidt J et al (2013) Preclinical models of multiple myeloma: a critical appraisal. Expert Opin Biol Ther 13(Suppl 1):S111-123. https://doi.org/10.1517/14712598.2013. 799131

Sze JH, Raninga PV, Nakamura K et al (2020) Anticancer activity of a Gold(I) phosphine thioredoxin reductase inhibitor in multiple myeloma. Redox Biol 28:101310. https://doi.org/10.1016/j.redox. 2019.101310

Thomsen AR, Aldrian C, Bronsert P et al (2017) A deep conical agarose microwell array for adhesion independent three-dimensional cell culture and dynamic volume measurement. Lab Chip 18:179189. https://doi.org/10.1039/c7lc00832e

Udi $\mathbf{J}$ et al (2013) Potent in vitro and in vivo activity of sorafenib in multiple myeloma: induction of cell death, CD138-downregulation and inhibition of migration through actin depolymerization. In: Br J Haematol. https://pubmed.ncbi.nlm.nih.gov/23384035/. Accessed 5 Feb 2021

Varga C, Waldschmidt JM, Gandolfi S, Richardson PG (2020) Current antibody-based therapies for the treatment of multiple myeloma. Clin Adv Hematol Oncol HO 18:736-748
Voorhees PM, Manges RF, Sonneveld P et al (2013) A phase 2 multicentre study of siltuximab, an anti-interleukin- 6 monoclonal antibody, in patients with relapsed or refractory multiple myeloma. Br J Haematol 161:357-366. https://doi.org/10.1111/bjh.12266

Waldschmidt JM, Simon A, Wider D et al (2017) CXCL12 and CXCR7 are relevant targets to reverse cell adhesion-mediated drug resistance in multiple myeloma. Br J Haematol 179:36-49. https://doi. org/10.1111/bjh.14807

Wang J, Wang J, Lopez E et al (2019) Repurposing auranofin to treat TP53-mutated or PTEN-deleted refractory B-cell lymphoma. Blood Cancer J 9:95. https://doi.org/10.1038/s41408-019-0259-8

Yaccoby S (2010) Osteoblastogenesis and tumor growth in myeloma. Leuk Lymphoma 51:213-220. https://doi.org/10.3109/10428 190903503438

Zdzisińska B, Roliński J, Piersiak T, Kandefer-Szerszeń M (2009) A comparison of cytokine production in 2-dimensional and 3-dimensional cultures of bone marrow stromal cells of multiple myeloma patients in response to RPMI8226 myeloma cells. Folia Histochem Cytobiol 47:69-74. https://doi.org/10.2478/v10042-009-0015-1

Zlei M, Egert S, Wider D et al (2007) Characterization of in vitro growth of multiple myeloma cells. Exp Hematol 35:1550-1561. https://doi.org/10.1016/j.exphem.2007.06.016

Publisher's Note Springer Nature remains neutral with regard to jurisdictional claims in published maps and institutional affiliations. 